\title{
Aprovisionamento Automático de Circuitos Ópticos via Plano de Controle GMPLS aplicado a uma Rede Óptica Reconfigurável Baseada em ROADMs
}

\author{
Giovanni C. Santos, Fábio D. Santos e Júlio. C. R. F. Oliveira
}

\begin{abstract}
Resumo-O aprovisionamento automático de circuitos ópticos via plano de controle GMPLS é demonstrado experimentalmente em um cenário de uma rede óptica reconfigurável baseada em ROADMs de grau 2. Utilizou-se algoritmos que desempenham de forma automática a descoberta e disseminação da topologia, o cálculo de rota, a escolha do comprimento de onda a ser transmitido, e a sinalização entre os elementos da rede óptica. $O$ plano de controle GMPLS desenvolvido propiciou o aprovisionamento de circuitos ópticos em toda a rede de maneira rápida, eficaz e confiável, através do controle das chaves ópticas que compõem os ROADMs.
\end{abstract}

Palavras-Chave-Redes Ópticas, Plano de Controle, GMPLS.

\begin{abstract}
The provisioning of optical circuits using GMPLS control plane is experimentally demonstrated in a reconfigurable optical networks based in 2-Degree ROADMs. Based on algorithms that calculate in an automatic way parameters like topology discovery and dissemination, route and wavelength assignments and signalization between network elements, the developed GMPLS control plane enable fast, efficient and reliable provisioning of optical circuits at all optical network, controlling ROADM optical switches.
\end{abstract}

Keywords-Optical amplifiers, optical networks, automatic gain control.

\section{INTRODUÇÃO}

Através do advento das redes ópticas com roteamento de comprimento de onda (canais), os sistemas de comunicações vêm apresentando um contínuo e significativo aumento em sua capacidade de transmissão, baseado na otimização da infraestrutura atual de fibras ópticas disponíveis nos backbones. Neste tipo de rede, o aprovisionamento, a proteção e a restauração de caminhos ópticos são processos dinâmicos e automáticos. Estas funções são realizadas através de elementos capazes de promover a inserção e remoção de sinais em determinados pontos da rede, proporcionando um elevado grau de reconfigurabilidade da mesma[1].

$\mathrm{Na}$ implementação de uma rede óptica com multiplexação de comprimentos de onda (redes WDM), o aprovisionamento e a reconfiguração das rotas que a compõem representam uma necessidade fundamental. No âmbito das redes metropolitanas, onde os serviços de transporte de alta capacidade são voltados para as grandes áreas de negócios, estas necessidades exibem custos excessivos. A maior parte destes custos deve-se à configuração manual da rede, normalmente realizada através

Giovanni C. Santos, Fábio D. Santos e Júlio. C. R. F. Oliveira, Diretoria de Redes de Telecomunicações; Fundação CPqD, Campinas, Brasil. Emails: gcuriel; fabios; julioc@cpqd.com.br. de OADM's (Optical Add/Drop Multiplexers) fixos. Assim, faz-se necessária a reserva de comprimentos de onda (em anéis DWDM metropolitanos e de acesso) a custos bem menores que os oferecidos pelas tecnologias atualmente disponíveis.

Os multiplexadores ópticos deriva/insere reconfiguráveis ROADM's (Reconfigurable Optical Add/Drop Multiplexers) se apresentam como a principal solução para reconfiguração remota e automática na camada óptica[2]. Atualmente, possuem uma vasta planta instalada, elevando significativamente o nível de reconfigurabilidade das redes ópticas de nova geração (ou redes ópticas ágeis, como também são conhecidas).

A reconfigurabilidade trazida pelos ROADM's torna necessária a adequação de outros dispositivos da camada óptica, como os Amplificadores Ópticos (EDFA's) - que exibem forte dependência do ganho com a potência de entrada -, equalizadores de potência, bloqueadores de comprimento de onda, transponders sintonizáveis, entre outros. O elevado grau de liberdade proporcionada às redes ópticas pelos elementos de reconfiguração (ROADM's e Cross-Connects) torna cada vez mais complexo o processo de otimização de alocação dos circuitos ópticos a serem utilizados.

No cenário de redes em malhas, compostas majoritariamente pela interconexão de anéis, torna-se impraticável a escolha manual das rotas a serem habilitadas, levando em conta os requisitos de camada física necessários. Cada nó dos anéis pode ser composto por diversos comprimentos de onda (inclusive sintonizáveis). Também existem amplificadores com ganho controlado, além de diferentes atenuações entre os enlaces que os interligam, podendo ser limitados por efeitos não lineares (FWM, SBS, SPM, entre outros).

Assim, torna-se imprescindível que todas estas limitações sejam consideradas no processo de habilitação de uma rota de forma automatizada, para a adequada operação da rede. No cenário ideal, o operador precisa oferecer apenas a origem e destino da rota (e possivelmente informações adicionais de Engenharia de Tráfego). A partir daí, algoritmos capazes de extrair a topologia e considerar as limitações encontradas calculam a melhor rota entre estes dois pontos.

Com o intuito de automatizar o processo de habilitação de circuitos em redes ópticas reconfiguráveis, é demonstrada a implementação de um plano de controle GMPLS (Generalized Multiprotocol Label Switching) capaz de automatizar o aprovisionamento de circuitos, fazendo uso de algoritmos de descoberta de topologia, sinalização e cálculo de rota. Na seção II, uma descrição geral sobre o GMPLS é apresentada; na seção III, o plano de controle GMPLS desenvolvido é 
apresentado; na seção IV, são apresentados os resultados obtidos na demonstração experimental de aprovisionamento automático baseado em um testbed de uma rede reconfigurável composta por ROADMs de grau 2. Por fim, na seção V são apresentadas as conclusões.

\section{GMPLS}

O GMPLS é uma evolução do MPLS (Multiprotocol Label Switching). Enquanto o MPLS foi desenvolvido com o intuito de gerenciar redes baseadas em pacotes, o GMPLS apresenta uma novo paradigma de controle. Neste, a rede é representada por um conjunto de camadas, cada uma responsável por trabalhar com um determinado protocolo (pacotes, WDM, comutação de fibras)[3]. A integração destas camadas oferece uma visão unificada e transparente da topologia.

O conjunto de protocolos GMPLS surgiu a partir de uma necessidade não satisfeita pelo MPLS: a reconfigurabilidade dinâmica da rede. Atualmente, a maioria das redes ópticas são estruturadas em anéis, o que torna a configuração dos elementos (até certo ponto) relativamente simples. Entretanto, o desenvolvimento das tecnologias de rede aponta para arquiteturas em malha, nas quais é exigida uma inteligência gerenciadora dos elementos constituintes da topologia. Este gerenciador deve prover de maneira rápida e eficiente condições para que rotas sejam estabelecidas entre dois pontos da rede, com proteção contras falhas e reconfigurabilidade rápida.

Questões relacionadas aos serviços disponibilizados pelas empresas provedoras também acabaram por impulsionar o desenvolvimento do GMPLS. Entre elas, destacam-se o oferecimento de larguras de banda dedicadas a um determinado tipo de serviço (transmissão de vídeo, por exemplo) e a necessidade de rotas protegidas contra falhas de qualquer natureza. Essas características podem ser obtidas através dos conceitos de Engenharia de Tráfego suportados por esse conjunto de protocolos[4].

Estruturalmente, o GMPLS divide-se em módulos. Cada um desenvolve uma ação na rede, de acordo com suas características. Existem três módulos principais dentro da arquitetura. $\mathrm{O}$ primeiro diz respeito à descoberta da topologia da rede, essencial para que qualquer ação de controle seja desenvolvida. $\mathrm{O}$ segundo trabalha com o cálculo de rotas entre os pontos de origem e destino; e o terceiro é responsável pela sinalização destas rotas entre os elementos intermediários e nos próprios clientes de origem e destino.

Existe no GMPLS uma divisão estratégica da rede em planos. Estes planos são independentes entre si, e juntos compõem a rede em sua totalidade. O Plano de Dados é a estrutura física de transmissão de dados na rede. Ele é composto pelos equipamentos de comutação (ROADM's, roteadores, switches), além de amplificadores ópticos, analisadores de espectro, transponders, entre outros. É neste plano que são efetivamente executadas as decisões a respeito do controle da rede (por exemplo, chaveamento de portas ópticas ou a ativação ou desativação de transponders).

O Plano de Controle é composto por elementos capazes de decidir sobre quais estratégias de roteamento serão utilizadas para o bom funcionamento da rede. Estes elementos (geral- mente computadores) se relacionam diretamente com integrantes do Plano de Dados, embora existam alguns casos em que não há interação - interfaces com clientes, por exemplo. Estes computadores contém instâncias dos blocos nos quais estão divididos o GMPLS, e são capazes de trabalhar em conjunto para determinar rotas quando solicitadas, já que conhecem a quem estão conectados, fornecendo uma visão estrutural completa da rede, conforme pode ser observado na Figura 1.

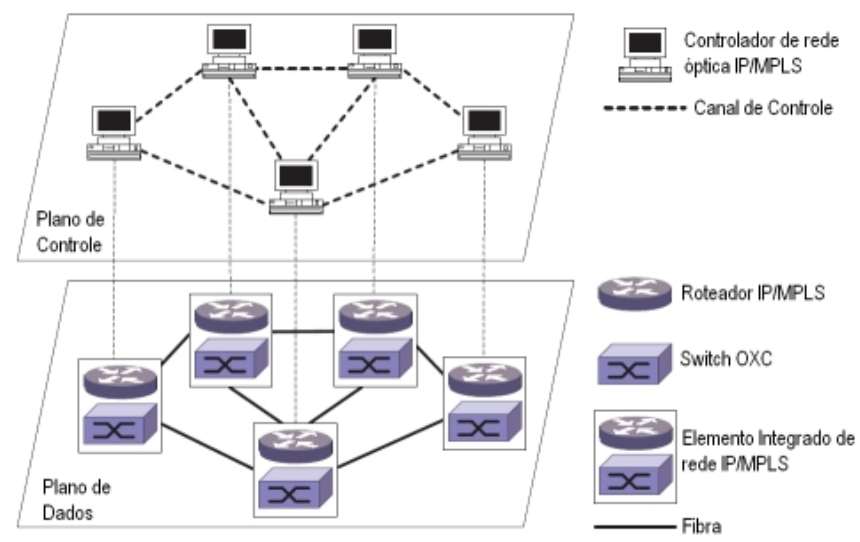

Fig. 1. Representação da divisão em Plano de Dados e Plano de Controle das redes ópticas reconfiguráveis controladas via GMPLS[5].

\section{Plano de Controle Desenvolvido}

O Plano de Controle desenvolvido foi dividido em módulos, cada um com uma função bem definida. São eles: Elemento de Cálculo de Rota (ECR), Elemento de Manipulação de Requisições (EMR) e Elemento de Controle de Equipamentos (ECE).

O ECR é responsável por calcular uma rota para uma requisição específica, dada uma topologia e algumas restrições. Estas restrições podem incluir características de camada física, preferências por certas tecnologias de transporte de dados, entre outras. Além disso, deve levar em consideração as características expressas na requisição de caminho, como as informações de qualidade de serviço, capacidade de encaminhamento dos equipamentos das pontas e protocolo de payload. É dever deste módulo também obter as rotas de proteção relacionadas ao pedido feito.

O EMR tem a função de obter a topologia da rede controlada (seja através de algoritmos específicos para este fim, seja através de arquivos descritivos de configuração). Também cuida do gerenciamento e agendamento de requisições de criação de caminhos e aplicação de políticas de uso do ECR. Deve ser acessível pela interface com o cliente, já que é ele quem iniciará o pedido de criação de caminho. Contém o módulo de cálculo de rota, que é alimentado com informações que o EMR obtém da rede.

$\mathrm{O}$ ECE recebe as mensagens de controle (criação, manutenção, remoção de caminhos) geradas pelo EMR, e executa as devidas configurações nos equipamentos do Plano de Dados. Para isso, conta com rotinas que permitem a identificação de quais equipamentos estão conectados à rede. A partir deste reconhecimento, um conjunto de comandos 
específicos para cada fabricante é selecionado. Assim, as ordens de reserva e liberação de recursos podem ser enviadas a diferentes equipamentos contidos em um mesmo caminho.

$\mathrm{O}$ funcionamento da arquitetura também é dividido em passos seqüenciais, conforme as etapas de criação do caminho. Inicialmente, é necessário detectar a topologia presente. Para isso, foi escolhido o protocolo Open Shortest Path First com extensões de Engenharia de Tráfego (OSPF-TE). Para a troca de mensagens de controle entre os controladores de equipamento, foi usado o protocolo Resource Reservation Protocol, também com extensões para Engenharia de Tráfego (RSVP-TE). Este último protocolo foi modificado para que fosse possível o controle dos ROADM's, incluindo aí os comprimentos de onda disponíveis no equipamento nas mensagens de criação do caminho óptico. Para a comunicação entre a interface com o cliente e o EMR, foi criado um novo protocolo não padronizado.

A configuração dos equipamentos foi feita usando o próprio protocolo já presente nos mesmos. Para que isso fosse possível, foi construído um módulo de configuração do ROADM que informa os dados necessários para sua configuração.

Para a implementação do Plano de Controle e do testbed, algumas características do sistema foram definidas. A conexão entre os elementos foi feita usando interfaces Ethernet; foram utilizados computadores industriais da fabricante VIA com sistema operacional Linux (distribuição Fedora Core 6). Os softwares do Plano de Controle foram escritos em $\mathrm{C} / \mathrm{C}++$, e empregaram-se ROADMs de tecnologia PLC (Planar Lightwave Circuit) no Plano de Dados. A topologia usada no testbed é a mostrada na Figura 2.

\section{Provisionamento Automático de Circuitos ÓPTICOS (DEMONSTRAÇÃO EXPERIMENTAL)}

A partir da estruturação física e lógica dos elementos da rede, inicia-se o processo de criação de um caminho óptico solicitado por um cliente. Este (CL1), acessando a interface fornecida, configura os parâmetros da requisição. Estes são constituídos pelos nós inicial e final do caminho, identificadores, largura da banda reservada, além de características dos equipamentos dos pontos terminais do caminho.

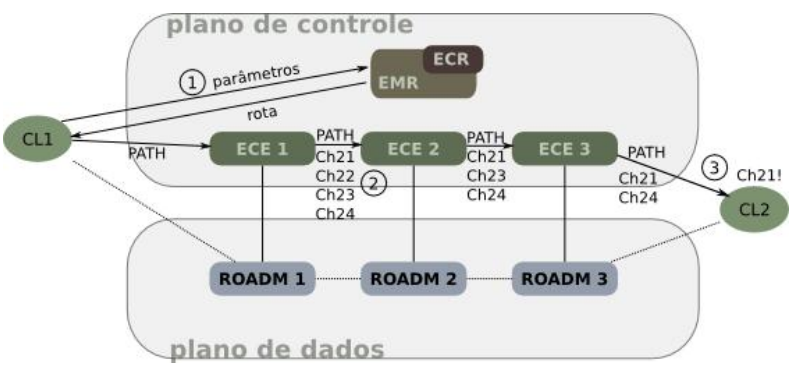

Fig. 2. Esquema de criação de um caminho óptico em uma rede reconfigurável controlada via GMPLS: indicação das etapas percorridas pela mensagem PATH.

A interface transmite esta requisição para o EMR. Este verifica os parâmetros e os repassa ao ECR, que busca na sua base de dados topológica (já construída devido à troca de mensagens OSPF-TE) uma rota que atenda aos requisitos do pedido. O EMR, quando receber a rota do ECR, devolve a resposta à interface cliente, que iniciará o processo de sinalização, usando o protocolo RSVP (passo 1, indicado na Figura 2). Um exemplo de resultado desta primeira etapa pode ser vista na saída de log do processo RSVP:

16:09:14 received PATH for 10.5.1.6/2001, 83952906
16:09:14 new Session: 10.5.1.6/2001/83952906
16:09:14 Processando mensagem PATH
16:09:14 MPLS: explicit route for 10.5.1.6 is:
IP4:11.0.0.2/32, IP4:11.0.0.5/32, IP4:11.0.0.6/32
IP4:11.0.0.9/32, IP4:11.0.0.10/32, IP4:11.0.0.13/32
IP4:11.0.0.14/32

A interface cliente, recebendo a rota do EMR, envia a primeira mensagem de criação do caminho óptico (PATH) para o primeiro nó presente na mesma (ECE 1). Quando $\mathrm{o}$ processo em execução do ECE 1 recebe a mensagem, verifica qual equipamento está sob seu controle, constatando que se trata de um ROADM. Dessa forma, ele obtém os canais disponíveis neste equipamento, preenchendo o objeto LABEL_SET contido na mensagem. Este objeto é utilizado para cadastrar os canais disponíveis nos equipamentos para que, no momento da reserva efetiva do caminho, o canal escolhido esteja verdadeiramente livre. A saída de $\log$ a seguir é um exemplo desta etapa:

16:09:22 Processando mensagem PATH

16:09:22 Iniciando o processamento do ERO

16:09:22 Rota encontrada do OSPF: ID: 10.4.1.162, inPort: 30 , outPort 101

16:09:22 ECE: SNMP: Tentando obter vendedor do equipamento...

16:09:25 ECE: SNMP: Vendedor reconhecido: ROADM 16:09:25 ECE: SNMP: Vendedor reconhecido: CPqD 16:09:25 ECE: Reiniciando valores de rotulos para MPLS

16:09:25 ECE: SNMP: Criando controlador para o ROADM...

Os nós intermediários recebem esta mensagem com este objeto preenchido, e retiram dele aqueles comprimentos de onda que já estão de alguma forma indisponíveis para uso (passo 2). O nó final, ao receber a mensagem PATH, escolhe um comprimento de onda do conjunto restante e constrói uma mensagem de resposta para a criação do caminho (RESV) com o objeto LABEL (passo 3). O conteúdo deste objeto é o canal referente ao comprimento de onda escolhido.

Os outros nós, ao receberem a mensagem RESV, configuram o equipamento e retiram da lista de comprimentos de onda disponíveis o presente na mensagem (passo 4, conforme indicado na Figura 3). Assim, quando a mensagem RESV chega ao cliente que solicitou o caminho, este tem a certeza de que o caminho foi criado com sucesso. Em caso contrário, algum erro ocorreu no processo, sendo que o cliente recebe uma mensagem informando este estado. A configuração do equipamento no processamento da mensagem RESV pode ser conferida na seguinte mensagem de log: 


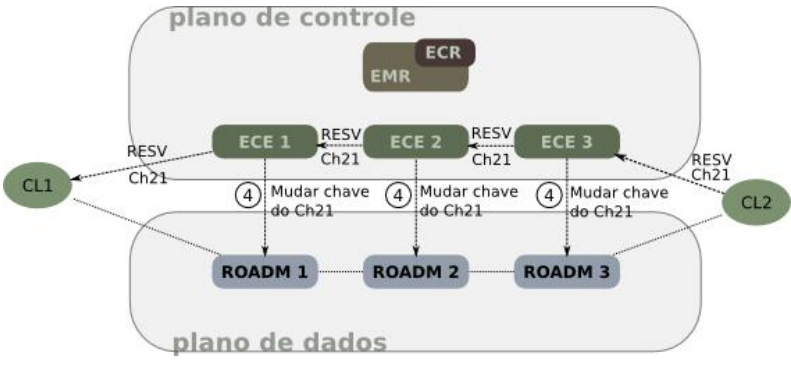

Fig. 3. Esquema de criação de um caminho óptico em uma rede reconfigurável controlada via GMPLS: indicação das etapas percorridas pela mensagem RESV.

16:09:40 Verificando rotulos(em um vetor de 40):

16:09:40 Canal 21

16:09:40 Rotulo encontrado, removendo da listas de rotulos disponiveis

16:09:40 ECE: Portas envolvidas 10030

16:09:40 ECE: No ROADM 10.4.1.164

16:09:40 Derivando canal 21

Para remover um caminho já criado, é necessário apenas o envio de uma mensagem de remoção de caminho óptico (PTEAR) para os nós que compõem o caminho. Esta informação está armazenada em uma sessão do RSVP-TE criada para o caminho (passo 5, localizado na Figura 4). Em cada nó para o qual a mensagem for transmitida, este liberará o canal referente ao caminho, enviando mensagens de configuração para o equipamento (passo 6).

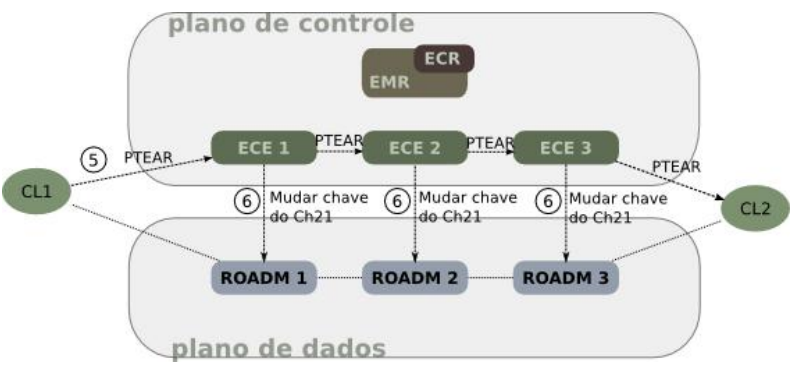

Fig. 4. Esquema de remoção de um caminho óptico em uma rede reconfigurável controlada via GMPLS: indicação das etapas percorridas pela mensagem PTEAR.

A configuração da remoção do caminho pode ser vista na saída de $\log$ a seguir:

16:12:13.005 gre12 sends MSG to 10.0.0.6

PTEAR 11 ttl:49 length:92

16:12:13.005 setting length in header field to 112

$16: 12: 13.005$ sending raw packet to 10.0 .0 .6 via 0.0 .0 .0 ip length: 112

16:12:13.005 Liberando lambda: 21

16:12:13.007 Rotulo NAO encontrado

Inserindo na lista de rotulos disponiveis

16:12:13.007 ECE: Liberando canal 21 do ROADM

Executando os passos apresentados nas Figuras 2 à 4, foi verificado experimentalmente a inserção e remoção de canais de forma automatizada, através do Plano de Controle GMPLS em desenvolvimento. As confirmações experimentais foram realizadas com o uso de analisadores de espectro óptico (OSA's), onde foi verificada a presença e remoção dos canais após as solicitações realizadas pelos clientes da rede (CL1). Os próximos passos, em construção, têm como objetivos a utilização de restrições de camada física no cálculo das rotas, assim como a construção de mecanismos de proteção na camada óptica.

\section{Conclusões}

O provisionamento de circuitos ópticos de forma automatizada foi demonstrado em um testbed de uma rede óptica reconfigurável baseada em ROADM's, através da utilização do Plano de Controle GMPLS em desenvolvimento no CPqD. Como apresentado, as fases de descoberta de topologia, cálculo de rota e sinalização são realizadas de forma automática, aprovisionando o canal (comprimento de onda) através do controle das chaves do ROADM.

O tempo médio de criação de um caminho foi de aproximadamente 40 segundos, e o de remoção próximo de 8 segundos. Estas operações normalmente levam, em redes cuja operação não é automática, algumas dezenas de dias para serem executadas. Ainda assim, os tempos alcançados por esta demostração poderão ser diminuídos ainda mais com a futura substituição do elemento de processamento existente no ROADM por um de maior capacidade.

Assim, foi demonstrada uma estrutura base de um Plano de Controle GMPLS que, no entanto, ainda encontra-se em construção, visando em um futuro próximo agregar funcionalidades de descoberta de topologia automática, proteção e algoritmos de RWA com restrições de camada física.

\section{REFERÊNCIAS}

[1] ELADA, L. et. al. 40-channel ultra-low-power compact PLC-based ROADM subsystem OFC 2006, Anaheim, Estados Unidos, 2006.

[2] METCONNEX. ROADM Architectures and Implementations Disponível em: http://www.metconnex.com/Products-page/roadm.htm. Acesso em: 12 Set. 2006

[3] E. Oki et al., Dynamic Multilayer Routing Schemes in GMPLS-Based $I P+$ Optical Networks IEEE Communications Magazine, pp 108-114, Jan. 2005.

[4] IEC, Generalized Multiprotocol Label Switching (GMPLS) The International Engineering Consortium, Web ProForum Tutorials, Mar. 2003.

[5] A. U. Fàbregas, Multi-Layer Survivability: Routing Schemes for GMPLS-Based Networks Department of Electronics, Computer Science and Automatic Control, Universitat de Girona, Espanha, Jul. 2006. 